\title{
ADVENTURES IN MACERAL SEPARATION
}

ANLCHMKP.-81225

Gary R. Dyrkacz

Chemistry Division

Argonne National Laboratory

9700 S. Cass Ave.

Argonne, Illinois 60439

\section{DISCLAIMER}

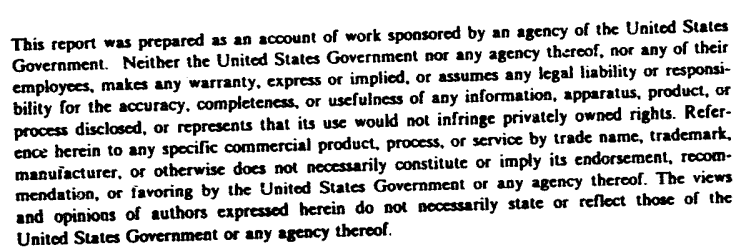

and opinions of authors expressed herein do
Unitod Stutes Government or any esency thereol.

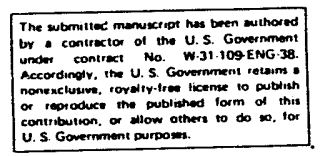

\section{argh}

DISTRIBUTION OF THIS DOCUMENT IS UNLIMITED

*This work was performed under the auspices of the Office of Basic Energy Sciences, Division of Chemical Sciences, U.S. Department of Energy, under contract number W-31-109-ENG-38. 
Gary R. Dyrkacz Chemistry Division,

Argonne National Laboratory

9700 S. Cass Ave

Argonne, Illinois 60439

\section{Introduction}

Understanding coal structure tasks the best researchers with the latest equipment. The problems start with the raw materials to produce coal - the profusion of plant tissues and minerals of the peat
swamps. The problems are further compounded by environmental processing of this raw material swamps. The problems are further compounded by environmental processing of this raw material
through a host ef biochemical, chemical and thermal transformations.' The resulting coal is then through a host ef biochemical, chemical and thermal transformations.'
physically and chemically heterogeneous, both in terms of the organic material and the mineral components. Just how heterogeneous is not even clear. Indeed, from the organic chemistry perspective, it is not even known whether any regular structure can even be assigned to coal.

Physically we divide coal into macroscopic or microscopic components called macerals as one Physically we dividic cols measure of the physcal heteroger living tissues such as spores or algae. More often the identity of the original material is uncertain.
Partly this is due to the accepted methods of preparing samples for microscopic observation, but Partly this is due to the accepted methods of preparing samples for microscopic observation, but
often the original peat is so extensively degraded that any remnants of the original physical structure are gone. ${ }^{2}$

Because macerals are the first level of heterogeneity that can be readily observed, separating them to obtain more pure species would appear to be worthwhile. This is true even for a single maceral type, such as vitrinite, which can still be heterogeneous. However, it is notable that maceral separations are not routinely done, even when fundamental structure studies are anticipated. This is colrat to my ore physical and chemical is contray separations of complex organic materials is a mandatory first stage in unraveling structures. One reason may have been a lack of maceral separation science. One of our goals has been to put maceral and, lately, submaceral separations on a more scientific basis.

The metho'ology of maceral separations can be broken down into several processes which are listed in Table 1. Each of these processes, when viewed in depth, is intertwined with complex and often little understood issues of coal's physical and chemical structure. As a result viable separation procedures are achieved mostly by guessing, faith and a lot of work. For efficient maceral separations to be realized, all these subprocesses must interlock in concert. We have been able to either thread our way through or skirt a number of the problems. However, there is still a largely unexplored vein of information within each area that is specifically important to maceral separations, unexplored vein of information within each area that is specifically important to maceral separations, and probably generally to coal science. A complete idea of where work is needed to improve
separations cannot be fully addressed here. Only a brief overview and flavor of where work stands can be given.

Discussion

\section{Liberation}

Coal constituents must be liberated from each other for optimal separation even to be possible. Without sufficient liberation some macerals may not be liberated at all. At best, they can only be enriched. Incomplete liberation also affects other constituents by limiting the pure materials that can be separated. This can potentially bias the type of material that is recovered as monomacera particles. For example. liptinites are usun!ly difficult to liberate cle:anly from vitinites. In density separations, these bimaceral particles will usually be found mixed in with low density monomaceral vitrinite particles. This vitrinite is different from any of the other vitrinite material. ${ }^{3}$ Eliminating this portion of material to obtain pure vitriniie can lead to differences in vitrinite properties that do not reflect the original coal.

Mechanical grinding has been the primary method for liberation. The small size of many maceral species means that the coal must be ground very fine - on the order of 10 microns. Fluid energy 
What is the appropriate stage to stop grinding? This question is not only important from the aspect Wf maceral separation, but can extend to how we perceive the chemical heterogeneity of coal of macer completely monomaceral. This goal is is opposed by the robsible below about 2 microns. In based on reflectance differences between addition, another limiting factor is reached in the submicron range ( $<0.5$ microns). Particles in this range can strongly interact with separation media giving quite different densities than larger particles with the same density.

The preceding demands of maceral liberation criticall $l_{j}$ depend on the maceral concept and macera identification. But what is the nature of chemical heterogeneity within even a single monomaceral idenifict If coal is ground to submicron sizes will new distinct particulate species appear? This panticle would suggest hat macerals the vitrinite will exhuif detiled substructures is so heterogeneous Alternatively, what if no further resolution occurs? This would be tre if coatis so herogeneous that it's structure varies on a nanometer scale, or if monomaceral particles are chemically homogeneous. These questions are not easy to answer, but point to a level of physical separation that has received only a small amount of attention.

\section{Demineralization}

The common minerals found in coal have densities from $\sim 1.5$ to 4 times that of the organic coa The con Thus a small amount of included mineral can have a large influence on maceral particle

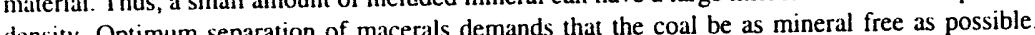
density. Optimum separic of Mechanical demineralization by density mas is Chemical demineralization using strong acids is the alternative, but whe the chemical structure of coal is still uncertain. Fonuitously, pyrie, which has a density of -4.5 $\mathrm{cm}^{-1}$ and is particularly difficult to remove chemically, is nearly completely liberated from the coal particles during fluid energy mill grinding.

\section{Wetting and Dispersion}

Mos maceral separtions are based on differences in maceral densities in liquid suspensions. If coal posticlion, then separation will be inefficient. There are two particles exten the media. related conpons ollisions over the 2. The paricles must remain isolated from one an separation time. Weting is a necessary conding for dispersion. Just whas govens the of a particular media for maceral particle dispersion is not clear. The result is that the dispersion remains in the realm of "shake-the-shelf " chemistry. An example of the importance of dispersion can be seen in Figure 1. This data is taken from a study of centrifugal sink/float separations for coa in a variety of commonly used suspension media. ${ }^{6} \mathrm{SI}$ is a separation index, which is a measure of the efficiency of separation. An SI value of 0 represents no separation relative to the original coal while 1 represents a complete disengagement of the float and sink material. A negative value is due while loction of float or sink phase found a material expected from a perfect found after separation, relative to the maxim separation. Thus, a value of 0 represents nc desired phase found, while I represents all the phase solated. Of the five media systems investigated, only two stood out: aqueous $\mathrm{CsCl} / \mathrm{Brij}-35$ and $\mathrm{Ca}\left(\mathrm{NO}_{3}\right)_{2} / \mathrm{Brij}-35$. (Brij-35 is the nonionic surfactant polyoxyethylene-23-lauryl ether.) Othe commonly used media, such as $\mathrm{ZnCl}_{2}$ or organic systems based on $\mathrm{CCl}_{4}$ were not as effective. Contrary to expectations, recycling material in aggregating media showed no further separation. Although Brij-35 seems to be a magic material that fixes all dispersion problems, it is no Separations in aqueous $\mathrm{ZnCl}, / \mathrm{Brij}-3$. solutions were only moderately better than $\mathrm{ZnCl}_{2}$ solutions Thus, the effect of the media can $b_{-}$subtle. Murh more work is needed to understand what drives the interactions.

Separation

The heart of maceral enrichment is the separation step. Sedimentation methods are the most come heration commonly used, particularly centrifugal sink/foat. A relative newcomer on the my type of sink/float separation. It has the highest density resolving ability of any maceral separation method, and 
functions both as a separation and characterization method. DGC methods are definitely the method of choice for optimum enrichment of macerals. However, DGC methods do have limitations on the amount of material than can be obtained in any single separation cycle. This can be overcome by first using sink/float methods to isolate desired density regions.

In some studies high density resolution of macerals may not be necessary. Thus, sink/float techniques will remain an alternate separation method or at least an adjunct to DGC separations. With this in mind, we have recently examined the process of centrifugal sink/float (S/F) separations to understand is limitations and provide guidance in its use. ${ }^{5.7}$. In addition, we have explored in to understand is lime have recenly examing detail a continuous flow centrifugation (CFC) technique hat allows much larger amounts of material to be separated in a shorter time than by simple centrifugal sink/loat. ${ }^{3}$ Both studies could not have been done without the resolving power and speed of analytical density gradient centrifugation (ADGC) methods. Each study required over two hundred separate ADGC analyses of float or sink phases.

Several general observations emerged from the sink/float studies. The effect of media has already been discussed. Figure 2 displays the ADGC results of a complete separation sequence on a single high volatile bituminous coal using $\mathrm{CsC} / \mathrm{Brij}-35$. The verical bars represent the nominal solution densities used for a hrom density to

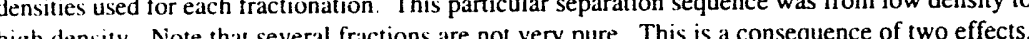
Nore First, even though a density separation may have a constant separation efficiency (i.e., the fraction of mass that will repon to the proper phase), the amount of contamination depends on the efficiency factor and the density distribution. Hence, separations made near large bands will be more contaminated than separations further from the m:an band. We were able to predict the purity of fractions based on the former effect with only moderate success. The second general observation is that float phases invariably contaminates the sink phase due to solution instabilities during the centrifugation process. Much more work is needed to optimize and predict the dynamics of such separations. In the course of this work, we also realized that the density interval of a fraction can separations. In also dramatically effect the ferity

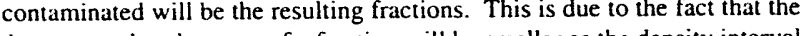
separation inefficiency is constant, but the mass of a fraction will be smaller as the density interval hetween separations decreases. DGC is not as affected by this phenomena; this is one reason for its high resolving power. Generally, the S/F separations produced fractions with purities $>60 \%$. Many fractions were routinely better than $80 \%$ pure.

Continuous flow centrifugation separations, another version of siniffloat separations, had very similar constraints to the simple centrifugal sink/float. Even the overall fraction purities were about the sane. CFC separations are carried out in a special centrifuge that allows the continuous removal of liquid fom the rolor. Float materal is entrained with the expelled liquid, while sink material is of liquid from retainedin the rotor (or $i$ can be used so that particle aggregation can be minimized. Single density separations with up to $300 \mathrm{~g}$ of coal can be accomplished within a day. This is far more than can be conveniently handled by simple centrifugal sink/float methods for finely ground coal. We believe that the efficiency of CFC separations can be substantially improved. For one the rotor dynamics appeared to be more complicated than generally believed. We suspect that there are regions within the separation section of the rotor where the particles are not subjected to the expected flow and centrifugal dynamics. Detailed studies of this device are needed to corroborate this. Modifications to the rotor may be necessary to increase the efficiency.

Isolation

Jolation is included in Table I mainly because of its impact on time for a complete separation cycle. The largest fraction of time in the separation of ultrafine coal is spent filtering and washing the separated samples. It is often wise to use membrane filters to avoid selective loss of fine coal, which further slows the filtration process.

Summary

Progress has been made in recent years in the science of maceral separation. However, there are many areas that can improved, and new areas to be investigated. The power of DGC to physically resolve miverals and submaceral species coupled with otier instrumental techniques is particularly attractive for defining the limits of coal heterogeneity as well as investigating the ajility of other separation methods. 


\section{Acknowledgement}

This work was performed under the auspices of the Office of Basic Energy Sciences, Division o Chemical Sciences, U. S. Department of Energy, under contract number W-31-109-ENG-38

\section{References}

1. Stach. E., Mackowsky, M.-Th., Teichmuller, M., Taylor, G. H., Chandra, D., Teichmuller, R. Murchinson, D.G., and Zienke, F., 'Stach's Textbook of Coal Petrology', 2nd Edn., Gebruder Borntraeger, Berlin, 1975.

Kroger, C., Erdol und Kohle Erdgas Petrochem., 1964, 17, 802-812.

3. Dyrkacz, G. R., Bloomquist, C. A. A., and Ruscic, L. R., Fuel, 1984, 63, 1166-1173.

4. Dyrkacz, G. R., Bloomquist, C. A. A., in preparation.

5. Dyrkacz, G. R., and Horwitz, E. P., Fuel, 1982, 61, 3 -12

6. Dyrkacz, G. R., Ruscic. L.R., and Fredericks, J., Energy and Fuets, 1992, 6, $720-742$.

7. Dyrkacz, G. R., Ruscic. L.R., ibid., 1992, 6, 743-752.

8. Dyrkacz, G. R., Bloomquist, C. A. A., ibid, 1992, 6, 357-374

9. Dyrkacz, G. R., Bloomquist, C. A. A.,ibid., 1992, 6, 375-386.

10.Dyrkacz, G. R., Bloomquist, C. A. A., Ruscic. L.R.,ibid., 1992, 7, 655-660

\section{Table 1. Coal Maceral Separation Subprocesses.}

\section{Liberation of constituents}

Demineralization

Wetting and dispersion of maceral particles

Separation

Isolation

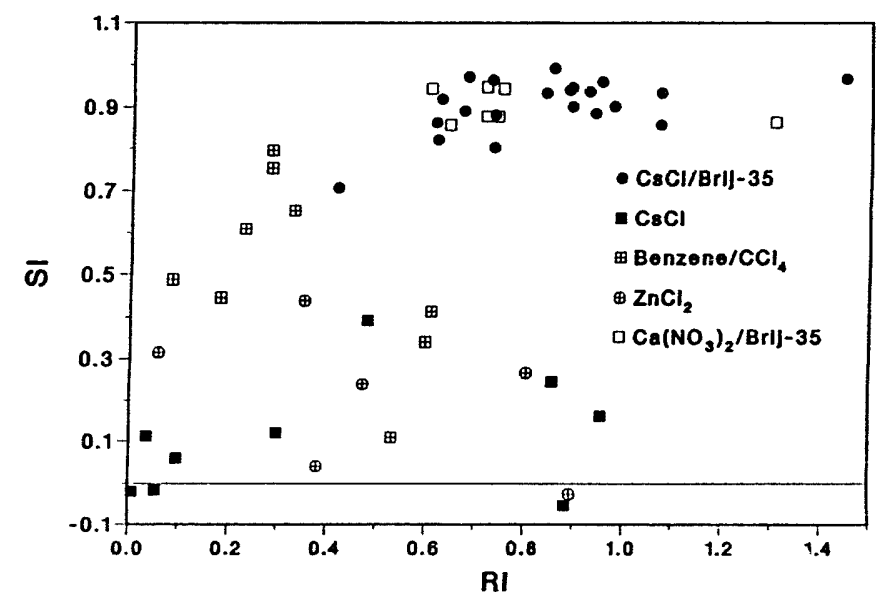

Figure 1. S/F Separation and recovery indices for float fractions from three high volatile bituminou 

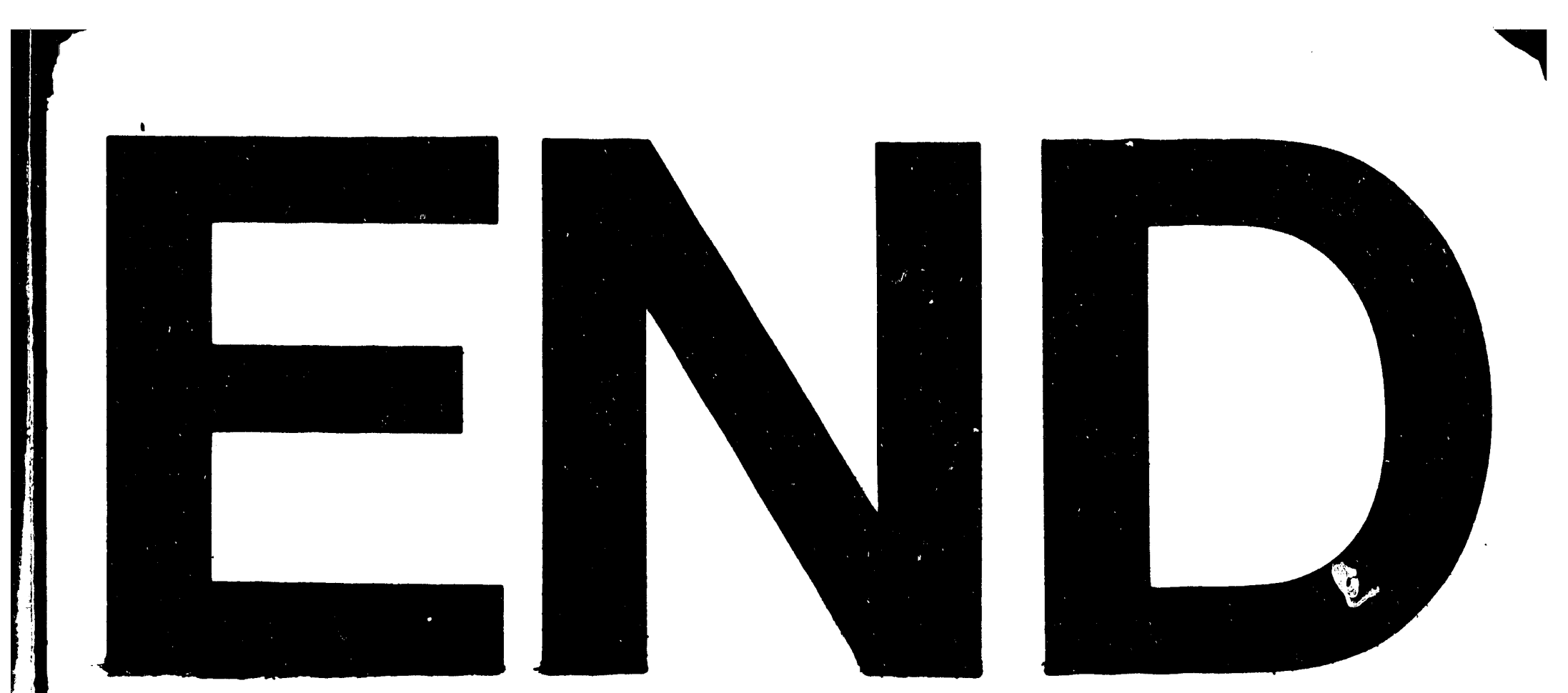

bestrom
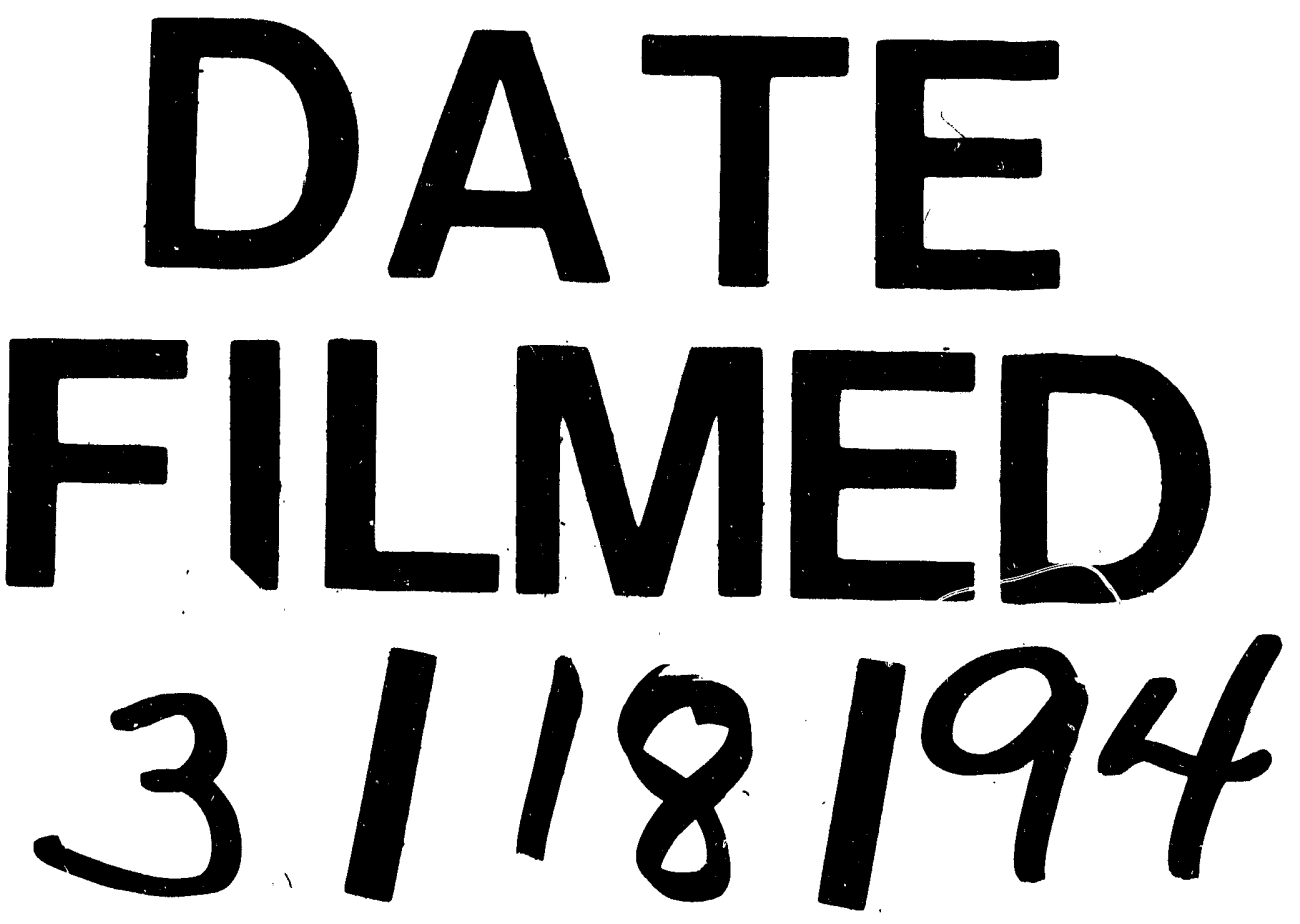


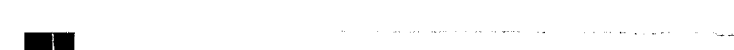

